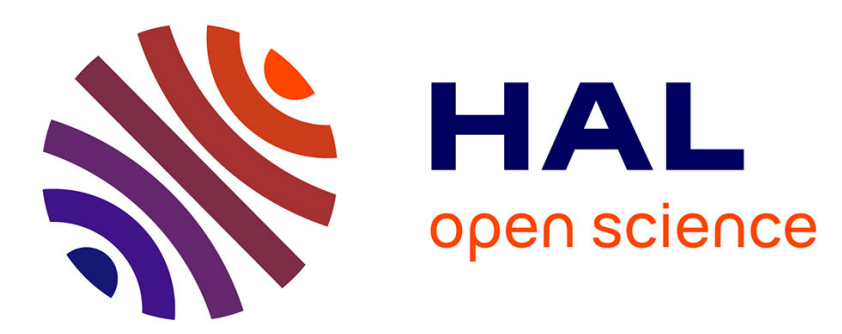

\title{
A micromechanics-based thermodynamic formulation of isotropic damage with unilateral and friction effects
}

\author{
Qi-Zhi Zhu, J.F. Shao, D. Kondo
}

\section{To cite this version:}

Qi-Zhi Zhu, J.F. Shao, D. Kondo. A micromechanics-based thermodynamic formulation of isotropic damage with unilateral and friction effects. European Journal of Mechanics - A/Solids, 2011, 10.1016/j.euromechsol.2010.12.005 . hal-00734535

\section{HAL Id: hal-00734535 \\ https://hal.science/hal-00734535}

Submitted on 23 Sep 2012

HAL is a multi-disciplinary open access archive for the deposit and dissemination of scientific research documents, whether they are published or not. The documents may come from teaching and research institutions in France or abroad, or from public or private research centers.
L'archive ouverte pluridisciplinaire HAL, est destinée au dépôt et à la diffusion de documents scientifiques de niveau recherche, publiés ou non, émanant des établissements d'enseignement et de recherche français ou étrangers, des laboratoires publics ou privés. 


\section{Accepted Manuscript}

Title: A micromechanics-based thermodynamic formulation of isotropic damage with unilateral and friction effects

Authors: Q.Z. Zhu, J.F. Shao, D. Kondo

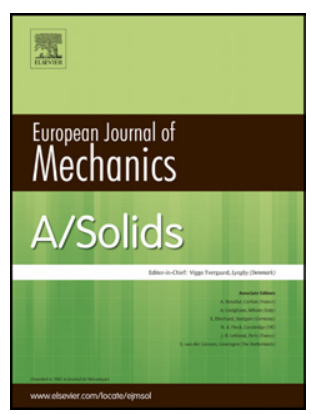

PII:

S0997-7538(10)00143-9

DOI:

10.1016/j.euromechsol.2010.12.005

Reference: EJMSOL 2660

To appear in: European Journal of Mechanics / A Solids

Received Date: 16 February 2010

Revised Date: 15 October 2010

Accepted Date: 18 December 2010

Please cite this article as: Zhu, Q.Z., Shao, J.F., Kondo, D. A micromechanics-based thermodynamic formulation of isotropic damage with unilateral and friction effects, European Journal of Mechanics / A Solids (2010), doi: 10.1016/j.euromechsol.2010.12.005

This is a PDF file of an unedited manuscript that has been accepted for publication. As a service to our customers we are providing this early version of the manuscript. The manuscript will undergo copyediting, typesetting, and review of the resulting proof before it is published in its final form. Please note that during the production process errors may be discovered which could affect the content, and all legal disclaimers that apply to the journal pertain. 


\title{
A micromechanics-based thermodynamic formulation of isotropic damage with unilateral and friction effects
}

\author{
Q.Z. Zhu ${ }^{\mathrm{a}, \mathrm{b}}$, J.F. Shao ${ }^{\mathrm{a}}$, D. Kondo ${ }^{\mathrm{a}}$ \\ ${ }^{a}$ Laboratoire de Mécanique de Lille-UMR CNRS 8107, Université de Sciences et Technologies Lille, \\ Cité Scientifique, Bd. Paul Langevin, 59655 Villeneuve d'Ascq Cedex, France \\ ${ }^{\mathrm{b}}$ Université Paris-Est, Laboratoire Modélisation et Simulation Multi-Echelle, MSME, UMR, 8208, \\ Cité Descartes, 77454, Marne la Valée Cedex 2
}

\begin{abstract}
This paper is devoted to micromechanical modeling of isotropic damage in brittle materials. The damaged materials will be considered as heterogeneous media composed of solid matrix weakened by isotropically distributed microcracks. The original contribution of the present work is to provide a proper micromechanical thermodynamic formulation for damage-friction modeling in brittle materials with the help of Eshelby's solution to matrix-inclusion problems. The elastic and plastic strain energy involving unilateral effects will be fully determined. The condition of microcrack opening-closure transition will be determined in both strain-based and stress-based forms. The effect of spatial distribution of microcracks will also be taken into account. Further, the damage evolution law is formulated in a sound thermodynamic framework and inherently coupled with frictional sliding. As a first phase of validation, the proposed micromechanical model is finally applied to reproduce basic mechanical responses of ordinary concrete in compression tests.
\end{abstract}

Key words: Homogenization; isotropic damage; friction; unilateral effects; thermodynamics; concrete.

\section{Introduction}

A proper description of inelastic behavior of cracked solids is essential for failure process modeling and stability analysis in many engineering applications. The present work is mainly devoted to one class of brittle geomaterials such as concrete and rocks. In such materials, the inelastic behavior is generally related to multiple mechanisms at different scales, such as void expansion or compaction, nucleation and propagation of microcracks, decohesion of matrix-aggregates interfaces, frictional sliding along weakness planes, phase transformation, and physical - chemical degradation. However, for a large range of stresses, damage due to nucleation and propagation of microcracks is considered as a key mechanism of inelastic deformation and failure process in such materials. Further, geomaterials may be subjected to both tensile

\footnotetext{
Email addresses: qizhi.zhu@univ-paris-est.fr (Q.Z. Zhu), jian-fu.shao@polytech-lille.fr (J.F. Shao), djimedo.kondo@univ-lille1.fr (D. Kondo).
} 
and compressive stresses and microcracks can be in open or closed states; there are strong unilateral effects on macroscopic responses. In the open state of microcracks, the inelastic deformation is directly related to displacement discontinuity which is characterized by normal opening and tangential shearing between two faces of microcracks. In the closed state, the inelastic deformation is generated by frictional sliding along crack faces. Moreover, the frictional sliding and microcrack growth leading to damage evolution are inherently coupled each other during irreversible processes involving the dissipation of energy and the evolution of microstructure (Wong, 1982; Fredrich et al., 1989).

In the past three decades, a number of macroscopic and phenomenological models have been developed for modeling coupled plastic deformation and induced damage in various materials, for instance Dragon and Mroz (1979), Ju (1989), Hayakawa and Murakami (1997), Shao (1998), Chiarelli et al. (2003), Shao et al. (2006) and others. Most of such models have been formulated within the framework of irreversible thermodynamics. It is generally assumed that the total free energy, used as the thermodynamic potential and expressed as a function of internal variables (plastic strain, damage variable, plastic hardening variable, etc...), can be decomposed into an elastic part and a plastic part, commonly termed as locked plastic work. Further, in view of some difficulties in mathematical determination, the locked plastic work is usually taken as a function of scalar-valued plastic hardening variable and damage variable. However, this assumption has never been theoretically and experimentally justified. Moreover, the mathematical description becomes quickly complex when it is needed to incorporate the so-called unilateral effects due to crack closure and interactions between microcracks. In order to overcome these shortcomings often revealed in classical phenomenological models, micromechanical damage models, mainly based on fracture mechanics and linear homogenization techniques, have been developed in parallel, for instance Andrieux et al. (1986), Gambarotta and Lagomarsino (1993), Krajcinovic (1996), Pensee et al. (2002), Zhu et al. (2008a,b). By taking into account the coupling between damage evolution and friction sliding, these models provide a better description of physical mechanisms at microscopic scale and their consequences on macroscopic responses, for instance, material softening due to localization and coalescence of microcracks, hysterestic phenomenon due to frictional sliding in cyclic loading, volumetric dilatancy related to asperity of crack faces, unilateral effects related to crack closure and irreversible strain after unloading, etc. However, most existing micromechanical models are limited to the analysis of solid matrix weakened by one single family of parallel microcracks and the case of interacting multi-families of microcracks is still a difficult task, especially when unilateral effects are involved simultaneously. Further, the basic concepts of linear fracture mechanics are generally used as the theoretical background without developing sound and rigorous up-scaling methods. Concerning the description of local frictional sliding along microcracks, most existing models do not take into account dilatancy-induced hardening effects due to passive crack opening during frictional sliding along closed rough microcracks. Finally, the local material softening related to the deterioration of internal friction and material cohesion has been usually neglected.

In the present work, a micromechanical model will be formulated by using sound homogenization methods and by taking into account the various difficult features mentioned above. Based on the recent works (Zhu et al., 2008a) on micromechanical modeling of anisotropic damage in brittle materials, the main emphasis of the present work is to develop a basic background for micromechanics-based thermodynamic formulation for the coupling between isotropic damage with unilateral effects and dissipative frictional sliding along microcracks. For the sake of clarity, we start by the simple case of unilateral damage evolution without friction sliding; the classical linear homogenization schemes (the dilute scheme (Eshelby, 1957), the Mori-Tanaka scheme (Mori and Tanaka, 1973) and the Ponte-Castaneda and Willis' estimate (Ponte-Castaneda and Willis, 1995)) will be adapted. The basic model is then logically extended by coupling the two dissipative mechanisms, i.e. damage evolution and friction sliding, for the case of closed microcracks. Compared with existing studies on this topic, the micromechanical model proposed here has the following specific features : i) development of a sound micromechanical basis via Eshelby's solutionbased homogenization method, with the consideration of spacial distribution of microcracks; ii) complete determination of the elastic and plastic strain energy involving unilateral effects; iii) determination of crack opening-closure transition condition in both strain-based and stress-based forms. It is also shown that the number of parameters in such micromechanical model is significantly reduced with respect to phenomenological ones.

Throughout the paper, the following notations on tensorial products of any second-order tensors $\boldsymbol{A}$ and 
$\boldsymbol{B}$ will be used : $(\boldsymbol{A} \otimes \boldsymbol{B})_{i j k l}=A_{i j} B_{k l}$. The tensor product of two vectors $\underline{a}$ and $\underline{b}$ is denoted $(\underline{a} \otimes \underline{b})_{i j}=a_{i} b_{j}$ and its symmetric part $(\underline{a} \stackrel{s}{\otimes} \underline{b})_{i j}=\frac{1}{2}\left(a_{i} b_{j}+a_{j} b_{i}\right)$. With the second rank identity tensor $\boldsymbol{\delta}$, the usually used fourth order isotropic tensors $\mathbb{I}$ and $\mathbb{J}$ are expressed in components form as $I_{i j k l}=\frac{1}{2}\left(\delta_{i k} \delta_{j l}+\delta_{i l} \delta_{j k}\right)$ and $J_{i j k l}=\frac{1}{3} \delta_{i j} \delta_{k l}$, respectively. The deviatoric operator $\mathbb{K}$ is then obtained by $\mathbb{K}=\mathbb{I}-\mathbb{J}$.

\section{General principles of upscaling method in cracked materials}

Consider the representative volume element (r.v.e.), occupying a domain $\Omega$ and having a boundary $\partial \Omega$, as a matrix-inclusion system, composed of the solid matrix with the elasticity tensor $\mathbb{C}^{s}$, assumed to be isotropic, and $N$ phases of inclusions with the stiffness tensor $\mathbb{C}^{c, r}, r=1, \ldots, N$, as shown in Fig.1a. The local mechanical behavior in $\Omega$ is assumed to be linear elastic : $\sigma(\underline{x})=\mathbb{C}(\underline{x}): \varepsilon(\underline{x})(\forall \underline{x} \in \Omega)$ with $\sigma(\underline{x})$ and $\varepsilon(\underline{x})$ as the local stress and strain fields, respectively. By making use of the superposition principle and taking the average of the local strain over $\Omega$, the general effective (homogenized) elasticity tensor is derived (Zaoui, 2002; Dormieux et al., 2006; Zhu et al., 2008a) :

$$
\mathbb{C}^{h o m}=\mathbb{C}^{s}+\sum_{r=1}^{N} \varphi^{r}\left(\mathbb{C}^{c, r}-\mathbb{C}^{s}\right): \mathbb{A}^{c, r}
$$

where $\varphi^{r}$ is the volume fraction representing the concentration of the $r$ th inclusion family. $\mathbb{A}^{c, r}$ is the averaged strain concentration tensor relating in a linear way the local strain $\varepsilon$ to the macroscopic uniform strain $\boldsymbol{E}$ on $\partial \Omega$, i.e. $\varepsilon=\mathbb{A}^{c}: \boldsymbol{E}$.
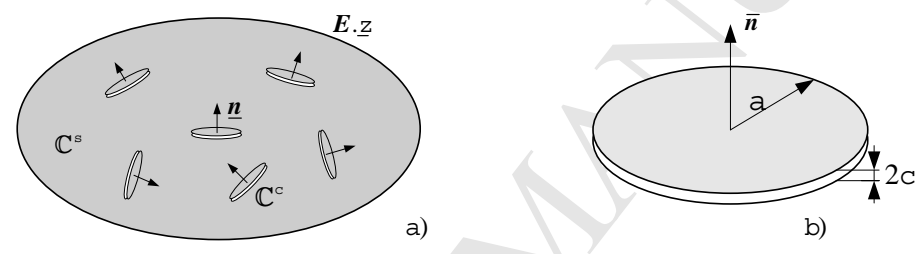

a)

b)

Fig. 1. a) R.v.e. of cracked solids; b) Schematic representation of a penny-shaped crack with aspect ratio $\epsilon=c / a$

The determination of homogenized elasticity tensor $\mathbb{C}^{\text {hom }}$ consists finally in evaluating the fourth order concentration tensor $\mathbb{A}^{c, r}$ for each phase. To this end, one can use the basic Eshelby's solution to the matrix-inclusion problem (Eshelby, 1957). For many engineering materials, it is useful to take into account both the influences of the shape of inclusions and those of their spatial distribution. The former is described by the fourth order Hill's tensor $\mathbb{P}_{\epsilon}^{r}$ which is linked to the Eshelby tensor by $\mathbb{S}_{\epsilon}=\mathbb{P}_{\epsilon}: \mathbb{C}^{s}$, while for the latter, another fourth order tensor $\mathbb{P}_{d}$ has been introduced (Ponte-Castaneda and Willis, 1995). For example, in the case of a spherical distribution of inclusions, $\mathbb{P}_{d}$ takes the form :

$$
\mathbb{P}_{d}=\frac{\alpha_{1}}{3 k^{s}} \mathbb{J}+\frac{\alpha_{2}}{2 \mu^{s}} \mathbb{K} ; \quad \text { with } \quad \alpha_{1}=\frac{3 k^{s}}{3 k^{s}+4 \mu^{s}} ; \quad \alpha_{2}=\frac{6\left(k^{s}+2 \mu^{s}\right)}{5\left(3 k^{s}+4 \mu^{s}\right)}
$$

where $k^{s}$ and $\mu^{s}$ denote respectively the bulk modulus and shear modulus of the solid matrix with $\mathbb{C}^{s}=$ $3 k^{s} \mathbb{J}+2 \mu^{s} \mathbb{K}$. The effective (homogenized) stiffness tensor for all the three considered homogenization schemes can be expressed in the following general form :

$$
\mathbb{C}^{\text {hom }}=\mathbb{C}^{s}-\left(\mathbb{I}+\mathbb{C}^{d}: \mathbb{P}_{d}\right)^{-1}: \mathbb{C}^{d}
$$

with

$$
\mathbb{C}^{d}=\sum_{r=1}^{N} \varphi^{r}\left(\mathbb{C}^{s}-\mathbb{C}^{c, r}\right):\left[\mathbb{I}-\mathbb{S}_{\epsilon}^{r}:\left(\mathbb{I}-\mathbb{S}^{s}: \mathbb{C}^{c, r}\right)\right]^{-1}
$$

The Mori-Tanaka estimate is obtained by setting $\mathbb{P}_{d}=\mathbb{P}_{\epsilon}$ for all families, equivalent to set $\alpha_{1}=\alpha_{2}=1$ in (2) whereas by taking $\mathbb{P}_{d}=0$, correspondingly, $\alpha_{1}=\alpha_{2}=0$, (3) reduces to the dilute homogenization 
scheme : $\mathbb{C}^{\text {hom }}=\mathbb{C}^{s}-\mathbb{C}^{d}$ implying that $\mathbb{C}^{d}$ can be interpreted as the modification by microcracks of dilute distribution to the matrix stiffness tensor $\mathbb{C}^{s}$.

\subsection{Application to cracked media}

Let's now consider cracked materials. A family of penny-shaped microcracks with a same normal can be approximated as flat ellipsoids characterized by its unit normal vector (orientation) $\underline{n}$ and the aspect ratio $\frac{c}{a}$, noted $\epsilon$, with $a$ being the average radius of cracks and $c$ the half-length of the small axis, as shown in Fig.1b. It is then possible to express mathematically the volume fraction $\varphi^{r}$ of the $r^{\text {th }}$ family of microcracks as follows :

$$
\varphi^{r}=\frac{4}{3} \pi a_{r}^{2} c_{r} \mathcal{N}_{r}=\frac{4}{3} \pi \epsilon \omega^{r}
$$

where $\mathcal{N}_{r}$ denotes the crack density (number of cracks per unit volume) of the $r^{t h}$ family, and $\omega^{r}=\mathcal{N}_{r} a_{r}^{3}$ is the damage parameter of microcracks, initially introduced by Budiansky and O'Connel (1976) and widely used as an internal variable in micromechanical models. For open cracks, the elasticity tensor $\mathbb{C}^{c}$ is taken as $\mathbb{C}^{c}=0$ by considering the cancellation of the stress on the crack faces. For closed frictionless planar cracks, it is shown in $\operatorname{Appendix}(\mathrm{A})$ that such cracks can be modeled as a fictitious material with the elasticity tensor $\mathbb{C}^{c}=\kappa \underline{n} \otimes \underline{n} \otimes \underline{n} \otimes \underline{n}$, reflecting the complete recovery of the elastic stiffness in the normal direction, in which $\kappa$ is positively definite. ${ }^{1}$ By the use of discrete damage parameters, the fourth order tensor $\mathbb{C}^{\omega}$ in (4) is rewritten as :

$$
\mathbb{C}^{\omega}=\sum_{r=1}^{N} \omega^{r} \mathbb{T}^{r}
$$

with $\mathbb{T}^{r}=\frac{4}{3} \pi \epsilon\left(\mathbb{C}^{s}-\mathbb{C}^{c, r}\right):\left[\mathbb{I}-\mathbb{S}_{\epsilon}^{r}:\left(\mathbb{I}-\mathbb{S}^{s}: \mathbb{C}^{c, r}\right)\right]^{-1}$. In the case of penny-shaped microcracks, the non vanishing components of the Eshelby tensor $\mathbb{S}_{\epsilon}$ are given in Mura (1987) and Nemat-Nasser and Hori (1993), and the expression in the Walpole's base is provided in Zhu et al. (2008a). Under the assumption of small aspect ratio $(\epsilon \ll 1), \mathbb{T}^{r}$ reads for open cracks

$$
\mathbb{T}^{r}=\frac{4}{3} \pi \mathbb{C}^{s}: \lim _{\epsilon \rightarrow 0} \epsilon\left(\mathbb{I}-\mathbb{S}_{\epsilon}^{r}\right)^{-1}
$$

and for closed cracks the expression $\mathbb{T}^{r}=\frac{4}{3} \pi\left(\mathbb{C}^{s}-\mathbb{C}^{c, r}\right): \lim _{\epsilon \rightarrow 0} \epsilon\left[\mathbb{I}-\mathbb{S}_{\epsilon}^{r}:\left(\mathbb{I}-\mathbb{S}^{s}: \mathbb{C}^{c, r}\right)\right]^{-1}$ is proved to be equivalent to the form :

$$
\mathbb{T}^{r}=\frac{4}{3} \pi \mathbb{C}^{s}: \lim _{\epsilon \rightarrow 0} \epsilon\left(\mathbb{I}-\mathbb{S}_{\epsilon}^{r}: \mathbb{E}^{4}\right)^{-1} .
$$

with $\mathbb{E}^{4}$ given in Appendix(A).

Since we are here interested in isotropic case, for both open and closed state of microcracks, the spatial damage distribution is then assumed to be uniform in the representative volume $\Omega$. This means that the discrete orientation-dependent damage variable $\omega^{r}$ is identical for all families. Therefore, by removing the superscript, we will take $\omega$ as the global damage variable for all crack families. Under this isotropic simplification and by making integration over the surface of a semi-sphere, we obtain the isotropic version of the homogenized (effective) stiffness tensor $\mathbb{C}^{\text {hom }}$ for cracked solids :

$$
\mathbb{C}^{\text {hom }}=3 k^{\text {hom }} \mathbb{J}+2 \mu^{\text {hom }} \mathbb{K}
$$

where $k^{\text {hom }}$ and $\mu^{\text {hom }}$ are the effective bulk modulus and shear modulus, respectively. In order to describe unilateral effects due to cracks closure and then distinguish the elastic moduli between the open and closed

1 Noticing that in the Walpole's base $\mathbb{J}=\left(\frac{2}{3}, \frac{1}{3}, 0,0, \frac{1}{3}, \frac{1}{3}\right)$, the special choice $\mathbb{C}^{c}=3 k^{s} \mathbb{J}$ by Deude et al. (2002), although lack of theoretic support, gives the same result in determination of the effective stiffness tensor. 
states,we introduce the following different superscripts for the elastic moduli, i.e. $k^{\text {hom,o }}$ and $\mu^{\text {hom,o }}$ for the open cracks and $k^{h o m, c}$ and $\mu^{h o m, c}$ for the closed ones, which are given as :

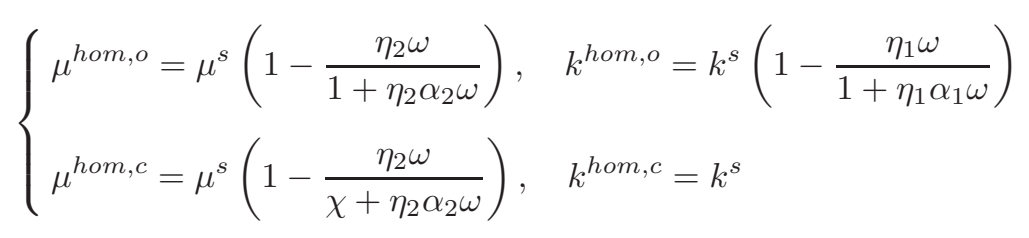

with $\eta_{1}=\frac{16\left(1-\left(\nu^{s}\right)^{2}\right)}{9\left(1-2 \nu^{s}\right)}, \eta_{2}=\frac{32\left(1-\nu^{s}\right)\left(5-\nu^{s}\right)}{45\left(2-\nu^{s}\right)}$ and $\chi=\frac{5-\nu^{s}}{3}$.

\subsection{Elastic damage formulation}

This section focuses on the constitutive formulation of damage model in the thermodynamic framework. The assumption of small strain and isothermal process is adopted in the present work. Generally, the formulation of damage model consists of the following three steps : i) choice of suitable damage variable(s) for the description of considered dissipative process; ii) determination of strain free energy from which it is derived the state laws and conjugated thermodynamic forces; and iii) finally, the choice of damage criterion for determining damage evolution. In the previous section, we have defined a scalar-valued global parameter $\omega$ as the internal damage variable and determined the effective elastic tensor via a standard homogenization procedure for cracked solids. The strain energy associated with the homogenized elastic tensor $\mathbb{C}^{\text {hom }}$, noted $W(\boldsymbol{E}, \omega)$, can be expressed as

$$
W(\boldsymbol{E}, \omega)=\frac{1}{2} \boldsymbol{E}: \mathbb{C}^{h o m}: \boldsymbol{E}=\frac{1}{2} k^{h o m}(\operatorname{tr} \boldsymbol{E})^{2}+\mu^{h o m} \operatorname{tr}\left(\boldsymbol{E}^{\prime} . \boldsymbol{E}^{\prime}\right)
$$

where $\boldsymbol{E}^{\prime}$ is the deviatoric part of the macroscopic strain tensor, $\boldsymbol{E}^{\prime}=\mathbb{K}: \boldsymbol{E}$. The macroscopic stress-strain relations are obtained by derivation of $W(\boldsymbol{E}, \omega)$ with respect to the total strain $\boldsymbol{E}$

$$
\boldsymbol{\Sigma}=\frac{\partial W}{\partial \boldsymbol{E}}(\boldsymbol{E}, d)=\mathbb{C}^{\text {hom }}: \boldsymbol{E}
$$

Similarly, the thermodynamic force associated with the damage variable $\omega$ is then obtained :

$$
F^{\omega}=-\frac{\partial W}{\partial \omega}(\boldsymbol{E}, \omega)=-\frac{1}{2} \frac{\partial k^{h o m}}{\partial \omega}(\operatorname{tr} \boldsymbol{E})^{2}-\frac{\partial \mu^{h o m}}{\partial \omega} \operatorname{tr}\left(\boldsymbol{E}^{\prime} . \boldsymbol{E}^{\prime}\right)
$$

with

$$
\frac{\partial k^{h o m}}{\partial \omega}=-\frac{\eta_{1}}{\left(1+\eta_{1} \alpha_{1} \omega\right)^{2}} k^{s}, \quad \frac{\partial \mu^{h o m}}{\partial \omega}=-\frac{\eta_{2}}{\left(1+\eta_{2} \alpha_{2} \omega\right)^{2}} \mu^{s}
$$

for open cracks, and

$$
\frac{\partial k^{h o m}}{\partial \omega}=0, \quad \frac{\partial \mu^{h o m}}{\partial \omega}=-\frac{\chi \eta_{2}}{\left(\chi+\eta_{2} \alpha_{2} \omega\right)^{2}} \mu^{s}
$$

for closed cracks.

When the damage criterion is based on the strain energy release rate, it should be a function of the driving force $F^{\omega}$ and the damage variable $\omega$, such that $f\left(F^{\omega}, \omega\right) \leq 0$. As usually performed, the damage evolution rate can be determined by using the standard normality rule :

$$
\dot{\omega}=\dot{\lambda}^{\omega} \frac{\partial f}{\partial F^{\omega}}
$$

where $\dot{\lambda}^{\omega}$ is commonly termed as damage multiplier and calculated by using the consistency condition $\dot{f}=0$, 


$$
\dot{f}=\frac{\partial f}{\partial \omega} \dot{\omega}+\frac{\partial f}{\partial \boldsymbol{E}}: \dot{\boldsymbol{E}}=\frac{\partial f}{\partial \omega} \frac{\partial f}{\partial F^{\omega}} \dot{\lambda}^{\omega}+\frac{\partial f}{\partial F^{\omega}} \frac{\partial F^{\omega}}{\partial \boldsymbol{E}}: \dot{\boldsymbol{E}}=0
$$

The insertion of (16) into (17) leads to the determination of $\dot{\lambda}^{\omega}$

$$
\dot{\lambda}^{\omega}=\frac{1}{H^{\omega}} \frac{\partial F^{\omega}}{\partial \boldsymbol{E}}: \dot{\boldsymbol{E}}
$$

with the damage hardening modulus $H^{\omega}=-\frac{\partial f}{\partial \omega}$. The incremental form of the macroscopic stress-strain relation in (12) is then expressed as $\dot{\boldsymbol{\Sigma}}=\mathbb{C}_{t}^{\text {hom }}: \dot{\boldsymbol{E}}$ with the fourth order symmetric tangent stiffness tensor $\mathbb{C}_{t}^{h o m}$ :

$$
\mathbb{C}_{t}^{h o m}=\mathbb{C}^{h o m}-\frac{1}{H^{\omega}} \frac{\partial F^{\omega}}{\partial \boldsymbol{E}} \otimes \frac{\partial F^{\omega}}{\partial \boldsymbol{E}}
$$

\section{Coupling between friction and unilateral damage}

The inelastic deformation due to displacement discontinuities generated a family of penny-shaped microcracks of normal $\underline{n}$, noted $\varepsilon^{c}$, takes the following general form :

$$
\varepsilon^{c}=\beta \underline{n} \otimes \underline{n}+\underline{\gamma} \stackrel{s}{\otimes} \underline{n}
$$

where the vector $\gamma$ is used to characterize the shear sliding along the crack planes whereas the scalar-

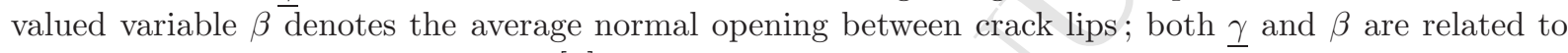
the local displacement discontinuity $[\underline{u}]$ by :

$$
\underline{\gamma}=\mathcal{N} \int_{\mathcal{S}^{+}}[\underline{u}] \cdot(\boldsymbol{\delta}-\underline{n} \otimes \underline{n}) d S ; \quad \beta=\mathcal{N} \int_{\mathcal{S}^{+}}[\underline{u}] \cdot \underline{n} d S
$$

The total macroscopic inelastic strain is obtained by the integration of $\varepsilon^{c}$ over the surface $\mathcal{S}^{+}$of a semi unit sphere :

$$
\boldsymbol{E}^{c}=\frac{1}{\Omega} \int_{\Omega^{c}} \varepsilon^{c} d V=\frac{1}{4 \pi} \int_{\mathcal{S}^{+}}(\beta \underline{n} \otimes \underline{n}+\underline{\gamma} \stackrel{s}{\otimes} \underline{n}) d S
$$

For the isotropic case and with the notation $\boldsymbol{\Gamma}=\frac{1}{4 \pi} \int_{\mathcal{S}^{+}} \underline{\underline{\gamma}} \stackrel{s}{\otimes} \underline{n} d S, \boldsymbol{E}^{c}$ can be decomposed into a mean part and a deviatoric part :

$$
\boldsymbol{E}^{c}=\frac{1}{3} \beta \boldsymbol{\delta}+\boldsymbol{\Gamma}, \quad \text { with } \beta=\operatorname{tr} \boldsymbol{E}^{c}
$$

In Section 2.2, we have discussed the elastic damage formulation within the thermodynamic framework for open and closed frictionless microcracks. Note that (12) can also be written as $\boldsymbol{\Sigma}=\mathbb{C}^{s}:\left(\boldsymbol{E}-\boldsymbol{E}^{c}\right)$. It is easy to obtain the explicit form of $\boldsymbol{E}^{c}$ as :

$$
\boldsymbol{E}^{c}=\left(\frac{\eta_{2} \omega}{1+\eta_{2} \alpha_{2} \omega} \mathbb{K}+\frac{\eta_{1} \omega}{1+\eta_{1} \alpha_{1} \omega} \mathbb{J}\right): \boldsymbol{E}
$$

for open cracks, with

$$
\beta=\frac{\eta_{1} \omega}{1+\eta_{1} \alpha_{1} \omega} \operatorname{tr}(\boldsymbol{E}), \quad \boldsymbol{\Gamma}=\frac{\eta_{2} \omega}{1+\eta_{2} \alpha_{2} \omega} \mathbb{K}: \boldsymbol{E}
$$

and

$$
\boldsymbol{E}^{c}=\boldsymbol{\Gamma}=\frac{\eta_{2} \omega}{\chi+\eta_{2} \alpha_{2} \omega} \mathbb{K}: \boldsymbol{E}
$$

for frictionless closed cracks with $\beta=0$ 
Remark 1 : The condition of unilateral contact on the frictionless crack faces is commonly written as

$$
\left[u_{n}\right] \geq 0 ; \quad \sigma_{n} \leq 0 ; \quad\left[u_{n}\right] \sigma_{n}=0
$$

where $\sigma_{n}=\boldsymbol{\sigma}:(\underline{n} \otimes \underline{n})$ is the normal component of the local stress tensor $\boldsymbol{\sigma}$ on the crack faces and $\left[u_{n}\right]=[\underline{u}] . \underline{n}$ is the normal component of the displacement jump vector $[\underline{u}]$ (crack aperture vector). Note that in the case of frictional closed cracks, the inelastic volumetric deformation described by the variable $\beta$ is mainly due to the rugosity of crack surfaces and passively induced by frictional sliding. Therefore, it is convenient to adopt $\sigma_{n}=0$ instead of $\left[u_{n}\right]$ (equivalently $\beta$ ) as the indicator of opening-closure transition.

Remark 2 : For frictional closed cracks, the explicit relations (24) and (25) do not hold any more due to energy dissipation in crack planes. In this case, an appropriate criterion for the study of frictional sliding is required, for instance the Coulomb-type criterion. In addition, the friction-induced inelastic deformation can be treated as plastic strain in the framework of the classic plasticity theory, and its evolution can be determined by following the normality rule within the thermodynamic framework.

\subsection{Macroscopic free energy based problem decomposition}

In most micromechanics-based damage models for brittle materials, the macroscopic free energy was generally composed of an elastic free energy purely caused by the solid matrix, and a blocked energy by self-equilibrated stresses due to the existence of displacement discontinuities (Gambarotta and Lagomarsino (1993), Pensee et al. (2002)), which implies that the solid matrix weakened by closed frictional microcracks exhibits the same effective elastic property as that in intact state, i.e. $\mathbb{C}^{\text {hom, } c}=\mathbb{C}^{s}$, and the damage dissipative process is only related to the blocked term. In this way, the closed frictional microcracks appear as quasi-statically frozen (blocked). Note that such static hypothesis has been widely adopted in micromechanical analysis for brittle materials (e.g. hard rocks and concrete).

According to the strain decomposition, i.e. $\boldsymbol{E}=\boldsymbol{E}^{e}+\boldsymbol{E}^{c}$, the initial homogenization problem $P$ can be decomposed into two sub-problems, as shown in Fig.2.

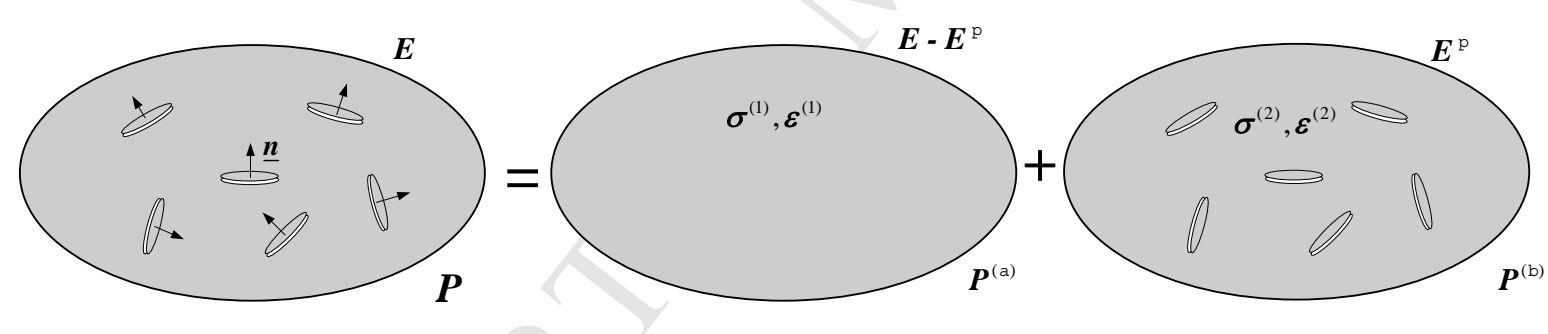

FIG. 2. Problem decomposition

In the sub-problem $P^{(1)}$, the stress field $\boldsymbol{\sigma}^{(1)}$ being uniform and statically admissible with the macroscopic stress and the stress field in the sub-problem $P^{(2)}$ being self-equilibrated, i.e. $\left\langle\boldsymbol{\sigma}^{(2)}\right\rangle_{\Omega}=0$, one obtains

$$
\Sigma=\sigma^{(1)}
$$

The strains in the two sub-problems obey to the homogenization principle over $\Omega$ and read

$$
<\varepsilon^{(1)}>_{\Omega}=\boldsymbol{E}-\boldsymbol{E}^{c}, \quad<\boldsymbol{\varepsilon}^{(2)}>_{\Omega}=\boldsymbol{E}^{c}
$$

In the sub-problem $P^{(1)}, \boldsymbol{\Sigma}$ is linked to the strain $\left(\boldsymbol{E}-\boldsymbol{E}^{c}\right)$ via the stiffness tensor $\mathbb{C}^{s}$ :

$$
\boldsymbol{\Sigma}=\mathbb{C}^{s}:\left(\boldsymbol{E}-\boldsymbol{E}^{c}\right)
$$

According to this decomposition, the global free energy $W$ is defined as 


$$
W=\frac{1}{2 \Omega} \int_{\Omega} \varepsilon^{(1)}(\underline{x}): \mathbb{C}^{s}(\underline{x}): \varepsilon^{(1)}(\underline{x}) d V+\frac{1}{2 \Omega} \int_{\Omega^{s}} \varepsilon^{(2)}(\underline{x}): \mathbb{C}^{s}(\underline{x}): \varepsilon^{(2)}(\underline{x}) d V
$$

The first term corresponds to the free energy in the sub-problem $P^{(1)}$ whereas the second one, noted $W^{(2)}$, is the contribution of the self-equilibrated stress field in the solid matrix $\boldsymbol{\sigma}^{(2)}\left(=\mathbb{C}^{s}: \varepsilon^{(2)}\right)$, which is due to the displacement discontinuities by microcracks. By making use of the self-equilibrating property, i.e. $\left\langle\boldsymbol{\sigma}^{(2)}>_{\Omega}=0\right.$, it is proved that the energy $W^{(2)}$ can be rewritten as :

$$
W^{(2)}=\frac{1}{2 \Omega} \int_{\Omega^{s}} \varepsilon^{(2)}(\underline{x}): \mathbb{C}^{s}: \varepsilon^{(2)}(\underline{x}) d V=-\frac{1}{2 \Omega} \int_{\Omega^{c}} \varepsilon^{(2)}(\underline{x}): \mathbb{C}^{s}: \varepsilon^{(2)}(\underline{x}) d V
$$

Under the isotropic assumption of the local stress field $\boldsymbol{\sigma}^{(2)}$ on cracks faces for all families and by using (30), the total strain energy $W$ takes finally the form :

$$
W=\frac{1}{2}\left(\boldsymbol{E}-\boldsymbol{E}^{c}\right): \mathbb{C}^{s}:\left(\boldsymbol{E}-\boldsymbol{E}^{c}\right)-\frac{1}{2} \boldsymbol{\sigma}^{(2)}: \boldsymbol{E}^{c}
$$

For complete determination of the total free energy $W$, it is necessary to establish the relation between $\boldsymbol{\sigma}^{(2)}$ and $\boldsymbol{E}^{c}$. To this end, we adapt the problem decomposition procedure used in Barthelemy et al. (2003) (see also Zhu et al. (2008a)) for the case that the solid matrix is weakened by one unique family of penny-shaped microcracks.

By applying the formulation procedure proposed in Zhu et al. (2008a) to the sub-problem $P^{(2)}$, i.e. taking $\boldsymbol{E}=\boldsymbol{E}^{c}$ and correspondingly $\boldsymbol{\Sigma}=0$, we obtain the following relation

$$
\boldsymbol{E}^{c}=-\left(\mathbb{I}-\mathbb{A}^{c}\right)^{-1}: \mathbb{A}^{c}: \mathbb{S}^{s}: \boldsymbol{\sigma}^{(2)}
$$

where $\mathbb{A}^{c}$ is termed as the global strain concentration tensor relating the inelastic strain $\boldsymbol{E}^{c}$ to the macroscopic strain $\boldsymbol{E}$ as defined in (24)

$$
\mathbb{A}^{c}=\frac{\eta_{2} \omega}{1+\eta_{2} \alpha_{2} \omega} \mathbb{K}+\frac{\eta_{1} \omega}{1+\eta_{1} \alpha_{1} \omega} \mathbb{J}
$$

The relation (34) can be also written in its inverted form :

$$
\boldsymbol{\sigma}^{(2)}=-\mathbb{C}^{b}: \boldsymbol{E}^{c}
$$

in which the explicit form of $\mathbb{C}^{b}=\left[\left(\mathbb{I}-\mathbb{A}^{c}\right)^{-1}: \mathbb{A}^{c}: \mathbb{S}^{s}\right]^{-1}$ reads :

$$
\mathbb{C}^{b}=3 k^{b} \mathbb{J}+2 \mu^{b} \mathbb{K}
$$

with the moduli $k^{b}=\frac{1+\eta_{1}\left(\alpha_{1}-1\right) \omega}{\eta_{1} \omega} k_{s}$ and $\mu^{b}=\frac{1+\eta_{2}\left(\alpha_{2}-1\right) \omega}{\eta_{2} \omega} \mu^{s}$. Insertion of (36) into (33) leads finally to the total free energy $W$ :

$$
W=\frac{1}{2}\left(\boldsymbol{E}-\boldsymbol{E}^{c}\right): \mathbb{C}^{s}:\left(\boldsymbol{E}-\boldsymbol{E}^{c}\right)+\frac{1}{2} \boldsymbol{E}^{c}: \mathbb{C}^{b}: \boldsymbol{E}^{c}
$$

It is seen that the total free energy $W$ is the sum of two terms, one representing to the elastic free energy of the solid matrix and another one, $\frac{1}{2} \boldsymbol{E}^{c}: \mathbb{C}^{b}: \boldsymbol{E}^{c}$, interpreted as the locked energy induced by closed frictional microcracks.

According to the superposition principle and the uniform character of the local stress field $\boldsymbol{\sigma}^{(1)}$ in the sub-problem $P^{1}$ as well as the self-equilibrium property of $\boldsymbol{\sigma}^{(2)}$ in the sub-problem $P^{2}$, the local stress field of the initial problem $P, \boldsymbol{\sigma}\left(=\boldsymbol{\sigma}^{(1)}+\boldsymbol{\sigma}^{(2)}\right)$ is expressed in the form :

$$
\boldsymbol{\sigma}=\boldsymbol{\Sigma}-\mathbb{C}^{b}: \boldsymbol{E}^{c}
$$

For a set of microcracks of normal $\underline{n}$, it is convenient to take the normal component of the local stress, i.e. $\sigma_{n}=\sigma:(\underline{n} \otimes \underline{n})$ as the indicator of the crack open/closed state. Under isotropic assumption, the 
global opening-closure transition condition $\mathcal{P}$ is then determined by making integration of the local one over the surface of a unit sphere $\mathcal{S}^{2}$ :

$$
\mathcal{P}=\frac{1}{4 \pi} \int_{\mathcal{S}^{2}} \boldsymbol{\sigma}:(\underline{n} \otimes \underline{n}) d S=\frac{1}{3} \sigma_{k k}=0 .
$$

Substitution of (23) and (37) into the above equation leads to the macroscopic stress-based transition condition

$$
\mathcal{P}(\boldsymbol{\Sigma})=\Sigma_{m}-k^{b} \beta=\Sigma_{m}-\frac{1+\eta_{1}\left(\alpha_{1}-1\right) \omega}{\eta_{1} \omega} k_{s} \beta=0,
$$

in which $\Sigma_{m}=\frac{1}{3} \Sigma_{k k}$ is the mean part of the macroscopic stress tensor, and accordingly the macroscopic strain-based condition reads

$$
\mathcal{P}(\boldsymbol{E})=E_{k k}-\frac{k^{s}+k^{b}}{k^{s}} \beta=E_{k k}-\frac{1+\eta_{1} \alpha_{1} \omega}{\eta_{1} \omega} \beta=0 .
$$

\subsection{Friction criterion and evolution law for closed cracks}

According to the second law of thermodynamics, the Clausius-Duhem inequality reads

$$
\boldsymbol{\Sigma}: \dot{\boldsymbol{E}}-\dot{W}=\left(\boldsymbol{\Sigma}-\mathbb{C}^{b}: \boldsymbol{E}^{c}\right): \dot{\boldsymbol{E}}^{c}-\frac{\partial W}{\partial \omega} \dot{\omega} \geq 0
$$

The thermodynamic force associated with the friction-induced plastic strain, noted $\boldsymbol{\sigma}^{c}$, is then given by

$$
\boldsymbol{\sigma}^{c}=-\frac{\partial W}{\partial \boldsymbol{E}^{c}}=\boldsymbol{\Sigma}-\mathbb{C}^{b}: \boldsymbol{E}^{c}
$$

Comparison between (39) and (44) shows that $\boldsymbol{\sigma}^{c}$ is exactly the local stress in the representative volume and according to the classic plasticity theory, plays the role of the driving force for frictional sliding on microcracks faces. Hence, the friction criterion should be function of appropriate invariants of $\boldsymbol{\sigma}^{c}$. For this, the fourth ordre isotropic tensors $\mathbb{J}$ and $\mathbb{K}$ are applied to $\sigma^{c}$ in order to extract its mean part and deviatoric part, noted $p^{c}$ and $\boldsymbol{S}^{c}$, respectively :

$$
\boldsymbol{\sigma}^{c}=\mathbb{J}: \boldsymbol{\sigma}^{c}+\mathbb{K}: \boldsymbol{\sigma}^{c}=\frac{1}{3}\left(\boldsymbol{\delta}: \boldsymbol{\sigma}^{c}\right) \boldsymbol{\delta}+\mathbb{K}: \boldsymbol{\sigma}^{c}
$$

The combination of $(30), 37)$ and (45) provides the mean part $p^{c}$ and deviatoric part $\boldsymbol{S}^{c}$ of the local stress $\sigma^{c}:$

$$
p^{c}=\frac{1}{3} \boldsymbol{\delta}: \boldsymbol{\sigma}^{c}=\Sigma_{m}-k^{b} \beta ; \quad \boldsymbol{S}^{c}=\mathbb{K}: \boldsymbol{\sigma}^{c}=\boldsymbol{S}-2 \mu^{b} \boldsymbol{\Gamma}
$$

Recall that $\Sigma_{m}$ and $\boldsymbol{S}$ are the commonly-used mean part and deviatoric part of the macroscopic stress $\boldsymbol{\Sigma}$, respectively. Based on this decomposition and by using the incremental form of (23), the inequality (43) is casted into the form :

$$
\boldsymbol{\Sigma}: \dot{\boldsymbol{E}}-\dot{W}=p^{c} \dot{\beta}+\boldsymbol{S}^{c}: \dot{\boldsymbol{\Gamma}}-\frac{\partial W}{\partial \omega} \dot{\omega} \geq 0
$$

from which is derived $p^{c}$ and $\boldsymbol{S}^{c}$ by differentiation of the free energy $W$ respectively with respect to the variables $\beta$ and $\boldsymbol{\Gamma}$ :

$$
p^{c}=-\frac{\partial W}{\partial \beta} ; \quad \boldsymbol{S}^{c}=-\frac{\partial W}{\partial \boldsymbol{\Gamma}}
$$

Based on these analysis, we will discuss two typical friction criteria, the Von Mises criterion and the generalized Coulomb friction criterion, in which use is made of the norm of the deviatoric stress $\boldsymbol{S}^{c}$ 


$$
\left\|\boldsymbol{S}^{c}\right\|=\sqrt{\boldsymbol{S}^{c}: \boldsymbol{S}^{c}}=\sqrt{\boldsymbol{\sigma}^{c}: \mathbb{K}: \boldsymbol{\sigma}^{c}}
$$

and the following friction-induced cumulated parameter $\gamma^{c}$ is introduced as the hardening variable :

$$
\gamma^{c}=\int \dot{\gamma}^{c}, \quad \dot{\gamma}^{c}=\sqrt{\dot{\boldsymbol{E}}^{c}: \mathbb{K}: \dot{\boldsymbol{E}}^{c}}=\sqrt{\dot{\boldsymbol{\Gamma}}: \dot{\boldsymbol{\Gamma}}}
$$

For simplicity, only the associated friction rules are discussed here.

\subsubsection{Generalized Coulomb friction criterion}

The generalized Coulomb type friction criterion is expressed as a linear function of the deviatoric stress $\boldsymbol{S}^{c}$ and the mean stress $p^{c}$ :

$$
g\left(\boldsymbol{\sigma}^{c}, \gamma^{c}\right)=\left\|\boldsymbol{S}^{c}\right\|+c_{f} p^{c}=\left\|\boldsymbol{S}-2 \mu^{b} \boldsymbol{\Gamma}\right\|+c_{f}\left(\Sigma_{m}-k^{b} \beta\right) \leq 0
$$

where $c_{f}$ denotes the friction coefficient. The evolution of the strain $\boldsymbol{E}^{c}$ is determined by

$$
\dot{\boldsymbol{E}}^{c}=\dot{\lambda}^{f} \frac{\partial g\left(\boldsymbol{S}^{c}, p^{c}\right)}{\partial \boldsymbol{\sigma}^{c}}=\dot{\lambda}^{f}\left(\boldsymbol{V}+\frac{1}{3} c_{f} \boldsymbol{\delta}\right)
$$

Correspondingly, the evolution rates of the components $\boldsymbol{\Gamma}$ and $\beta$ rae given by

$$
\dot{\boldsymbol{\Gamma}}=\dot{\lambda}^{f} \boldsymbol{V} ; \quad \dot{\beta}=c_{f} \dot{\lambda}^{f}
$$

The increment of the hardening variable $\gamma^{c}$ is determined by

$$
\dot{\gamma}^{c}=\sqrt{\dot{\Gamma}: \dot{\Gamma}}=\dot{\lambda}^{f}
$$

Inversely, we have $\dot{\boldsymbol{\Gamma}}=\dot{\gamma}^{c} \boldsymbol{V} ; \quad \dot{\beta}=c_{f} \dot{\gamma}^{c}$. In this way, the scalar hardening variable $\gamma^{c}$ is inherently involved in the above criterion.

\subsubsection{Yield criterion of Von Mises type}

Mathematically, the Von Mises type yield criterion is commonly expressed as :

$$
g\left(\boldsymbol{S}^{c}, \gamma^{c}\right)=\left\|\boldsymbol{S}^{c}\right\|-\kappa_{c}=\left\|\boldsymbol{S}-2 \mu^{b} \boldsymbol{\Gamma}\right\|-\kappa_{c}\left(\gamma^{c}\right) \leq 0
$$

The evolution rate of the inelastic strain $\boldsymbol{E}^{c}$ is then determined by

$$
\dot{\boldsymbol{E}}^{c}=\dot{\lambda}^{f} \frac{\partial g\left(\boldsymbol{S}^{c}\right)}{\partial \boldsymbol{\sigma}^{c}}=\dot{\lambda}^{f} \boldsymbol{V}
$$

where the factor $\dot{\lambda}^{f}$ is referred to as the non-negative multiplier and generally determined by the consistency condition $\dot{g}=0$. Comparison of (56) with the incremental form of (23),

$$
\dot{\boldsymbol{E}}^{c}=\frac{1}{3} \dot{\beta} \boldsymbol{\delta}+\dot{\boldsymbol{\Gamma}},
$$

allows the evolution rate of the variables $\boldsymbol{\Gamma}$ and $\beta$ to be derived for the Von Mises-type yield criterion

$$
\dot{\boldsymbol{\Gamma}}=\dot{\lambda}^{f} \boldsymbol{V} ; \quad \dot{\beta}=0
$$

Note that the criterion (55) does not take into account influences of confining pressures (or mean stress) on plastic deformation, which, however, is essential for geomaterials under compressive loading. Further, for an associated flow rule, this model is not able to predict inelastic volumetric deformation. 


\subsubsection{Verification of continuities at opening-closure transition}

From physical point of view, when any opening-closure transition of microcracks occurs, the stresses and free energy need to remain continuous although the material stiffness may suffer a strong discontinuity (jump). Therefore, a proper friction criterion should take into account this requirement of continuity. We first check the Coulomb friction criterion (51). When the opening-closure transition condition (41) (equivalently (42)) is verified, one has

$$
\Sigma_{m}-k^{b} \beta=0
$$

and

$$
E_{k k}-\frac{1+\eta_{1} \alpha_{1} \omega}{\eta_{1} \omega} \beta=0
$$

from (41) and 42), respectively.

Given $\left\|\boldsymbol{S}^{c}\right\| \geq 0$, the insertion of (59) into the Coulomb criterion (51) leads to $\boldsymbol{S}-2 \mu^{b} \boldsymbol{\Gamma}=0$, which can be given in strain-based form by

$$
\boldsymbol{E}^{\prime}-\frac{1+\eta_{2} \alpha_{2} \omega}{\eta_{2} \omega} \boldsymbol{\Gamma}=0
$$

We then derive from (60) and (61) the relations

$$
\beta=\frac{\eta_{1} \omega}{1+\eta_{1} \alpha_{1} \omega} E_{k k}, \quad \boldsymbol{\Gamma}=\frac{\eta_{2} \omega}{1+\eta_{2} \alpha_{2} \omega} \boldsymbol{E}^{\prime}
$$

Their insertion into (30) and (38) leads to the expressions of the macroscopic stress and the total free energy (11) in open state of microcracks, implying the simultaneous satisfaction of the continuity of stresses and free energy at the opening-closure transition.

However, according to the Von Mises criterion (55) which is independent of the local mean stress $p^{c}$, when the transition condition is verified, we have $\boldsymbol{S}-2 \mu^{b} \boldsymbol{\Gamma} \neq 0$ for the general case that $\kappa_{c}\left(\gamma^{c}\right) \neq 0$. Consequently, the continuities of stresses and free energy are generally not satisfied for this criterion.

\subsection{Coupling between damage and friction}

Based on the strain free energy (38), the thermodynamic force associated with the internal damage variable $\omega$, noted $F^{\omega}$, is derived by standard differentiation of the macroscopic free energy $W$ :

$$
F^{\omega}=-\frac{\partial W}{\partial \omega}=-\frac{1}{2} \boldsymbol{E}^{c}: \frac{\partial \mathbb{C}^{b}}{\partial \omega}: \boldsymbol{E}^{c}
$$

or in explicit form

$$
F^{\omega}=\frac{1}{2 \eta_{1} \omega^{2}} k^{s} \beta^{2}+\frac{1}{\eta_{2} \omega^{2}} \mu^{s} \boldsymbol{\Gamma}: \boldsymbol{\Gamma}
$$

It is seen that $F^{\omega}$ is independent of homogenization scheme and that damage propagation is totally caused by frictional sliding on cracks faces via the cumulation of the variables $\beta$ et $\Gamma$. When a strain energy release rate based damage criterion is used, the criterion $f\left(\boldsymbol{E}^{c}, \omega\right) \leq 0$ should be function of the friction-induced inelastic strain $\boldsymbol{E}^{c}$ and the damage variable $\omega$. Note that the moduli $k^{b}$ and $\mu^{b}$ in the fourth order tensor $\mathbb{C}^{b}$ are also function of the damage variable. In fact, the Coulomb criterion (51) can also be expressed in strain-based form :

$$
g\left(\boldsymbol{E}, \beta, \boldsymbol{\Gamma}, \gamma^{c}\right)=2 \mu^{s}\left\|\boldsymbol{E}^{\prime}-\frac{1+\eta_{2} \alpha_{2} \omega}{\eta_{2} \omega} \boldsymbol{\Gamma}\right\|+c_{f} k^{s}\left(\operatorname{tr} \boldsymbol{E}-\frac{1+\eta_{1} \alpha_{1} \omega}{\eta_{1} \omega} \beta\right) \leq 0
$$

When damage propagation and frictional sliding take place simultaneously, these two dissipative mechanisms will interfere each other in a strong manner in the sense of the determination of their evolution. 
In this case, one obtains the following system to be solved, which is constructed by the consistency conditions for damage $\dot{f}=0$ and for friction, $\dot{g}=0$ :

$$
\left\{\begin{array}{l}
\dot{f}=\frac{\partial f}{\partial \omega} \dot{\omega}+\frac{\partial f}{\partial \beta} \dot{\beta}+\frac{\partial f}{\partial \boldsymbol{\Gamma}}: \dot{\boldsymbol{\Gamma}}=0 \\
\dot{g}=\frac{\partial g}{\partial \omega} \dot{\omega}+\frac{\partial g}{\partial \beta} \dot{\beta}+\frac{\partial g}{\partial \boldsymbol{\Gamma}}: \dot{\boldsymbol{\Gamma}}+\frac{\partial g}{\partial \boldsymbol{E}}: \dot{\boldsymbol{E}}=0
\end{array}\right.
$$

Note also that $\dot{\boldsymbol{E}}^{c}=\dot{\lambda}^{f} \frac{\partial g}{\partial \boldsymbol{\sigma}^{c}}$ and $\dot{\omega}=\dot{\lambda}^{\omega} \frac{\partial f}{F^{\omega}}$. It follows the expression of the damage and plasticity multipliers :

$$
\left\{\begin{array}{l}
\dot{\lambda}^{f}=\frac{1}{H^{f w}} \frac{\partial g}{\partial \boldsymbol{E}}: \dot{\boldsymbol{E}} \\
\dot{\lambda}^{\omega}=-\frac{\frac{\partial f}{\partial \beta} c_{f}+\frac{\partial f}{\partial \boldsymbol{\Gamma}}: \boldsymbol{V}}{\frac{\partial f}{\partial d} H^{f w}} \frac{\partial g}{\partial \boldsymbol{E}}: \dot{\boldsymbol{E}}
\end{array}\right.
$$

with

$$
H^{f w}=\left[\frac{\partial g}{\partial \omega}\left(\frac{\partial f}{\partial \beta} c_{f}+\frac{\partial f}{\partial \boldsymbol{\Gamma}}: \boldsymbol{V}\right)-\frac{\partial f}{\partial \omega}\left(\frac{\partial g}{\partial \beta} c_{f}+\frac{\partial g}{\partial \boldsymbol{\Gamma}}: \boldsymbol{V}\right)\right] / \frac{\partial f}{\partial \omega}
$$

Accordingly, the tangential operator $\mathbb{C}_{t}^{\text {hom }}$ linking the macroscopic stress increment to the macroscopic strain increment, i.e. $\dot{\boldsymbol{\Sigma}}=\mathbb{C}_{t}^{\text {hom }}: \dot{\boldsymbol{E}}$, is now given as

$$
\mathbb{C}_{t}^{\text {hom }}=\mathbb{C}^{\mathrm{s}}-\frac{1}{H^{f w}} \frac{\partial g}{\partial \boldsymbol{E}} \otimes \frac{\partial g}{\partial \boldsymbol{E}}
$$

with $\frac{\partial g}{\partial \boldsymbol{E}}=2 \mu^{s} \boldsymbol{V}+c_{f} k^{s} \boldsymbol{\delta}$. $\mathbb{C}_{t}^{\text {hom }}$ possesses the major and minor symmetries since we have adopt an associated flow rule.

\section{First validations}

This section is devoted to show the performence of the proposed model in predicting main features of mechanical behaviors for quasi-brittle materials. A frist phase of validation is presented. Numerical simulations of a few typical laboratory tests on concrete materials are performed with the proposed model and compared with experimental data. We adopt here the Coulomb-type friction criterion defined in (51) or (65) and an associated flow rule for the evolution of inelastic strains $\boldsymbol{E}^{c}$. In addition, the following damage criterion is proposed :

$$
f\left(F^{\omega}, \omega\right)=F^{\omega}-\kappa_{0}-\frac{1}{c_{1}} \tan \left(\frac{\pi}{2} \frac{\omega-\omega_{0}}{\omega_{c}-\omega_{0}}\right)=0
$$

where $\kappa_{0}$ represents the initial damage threshold. For the sake of simplicity, its value is arbitrarily fixed at $\kappa_{0}=0 ; \omega_{0}$ and $\omega_{c}$ are initial and critical values of the damage variable, respectively; the term $\frac{1}{c_{1}} \tan \left(\frac{\pi}{2} \frac{\omega-\omega_{0}}{\omega_{c}-\omega_{0}}\right)$ characterizes damage hardening effect controlled by the parameter $c_{1}$.

In the present numerical simulations, $\omega_{0}$ is taken as a small positive value $\omega_{0}=0.001$ due to the lack of experimental data on the initial distribution of microcracks. Further, in compressive conditions, the dissipation mechanism is essentially related to frictional sliding along crack faces and the induced damage mainly affects the macroscopic shear modulus. It is thus reasonable to link the parameter $\omega_{c}$ to the degradation of macroscopic shear modulus $\mu^{\text {hom }}$ in (10). We derive from the condition $\mu^{\text {hom }} \geq 0$ :

$$
\omega \leq \frac{1}{\eta_{2}\left(1-\alpha_{2}\right)}
$$


which provides the critical value $\omega_{c}=\frac{1}{\eta_{2}\left(1-\alpha_{2}\right)}$. Consistently, the inequality (71) also leads to the nonnegative property of the modulus $\mu^{b}$ implying a continuously kinematic hardening of the yield function. In order to illustrate the range of $\omega_{c}$ for different homogenization schemes (the dilute scheme, the MoriTanaka scheme (M-T) and the Ponte-castaneda and Willis' estimate (PC-W)), we depict the evolution of the ratio $\frac{\mu^{\text {hom }}}{\mu^{s}}$ with damage variable $\omega$, for each scheme and for three values of Poisson's ratio of the solid matrix $\nu^{s}=0.15, \nu^{s}=0.20$ and $\nu^{s}=0.25$, as shown in Fig.3. The results show that the critical values of damage for the considered homogenization schemes are very different : this value is lower than 1.0 for the dilute scheme while higher than 1.0 for the scheme PC-W; as for the Mori-Tanaka estimate, there is no bound. Furthermore, it seems that the influence of Poisson's ratio on critical damage value is not significant.

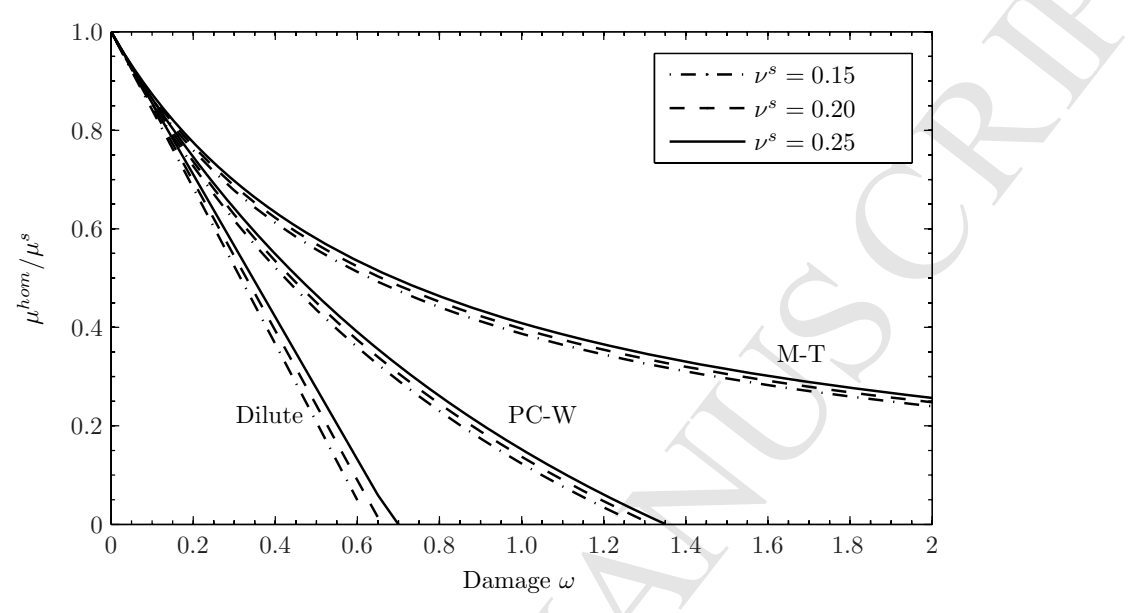

FIG. 3. Relative evolution with damage of the effective shear modulus for three homogenization schemes

\subsection{Numerical predictions for monotonic loading}

The proposed coupled damage-friction model is now applied to simulate two compression tests reported by Van Mier (1984) and performed on concrete materials under uniaxial loading and under plane-strain condition, respectively. Based on the discussions above, the proposed model contains four parameters : two elastic constants for initial (undamaged) state of material, $E^{s}$ and $\nu^{s}$, which may be determined from the linear part of stress-strain curves before the initiation of damage evolution, the friction coefficient $c_{f}$ involved in the Coulomb friction criterion as well as the damage parameter $c_{1}$ controlling the material softening. These two parameters have been calibrated by means of a simple compression test for the homogenization scheme proposed by the Ponte-castaneda and Willis (Ponte-Castaneda and Willis, 1995), due to the fact that among the considered homogenization schemes, only the PC-W one has the ability of properly taking into account the influences of interactions between microcracks. The parameter values used for numerical simulations are : $E^{s}=35000 \mathrm{MPa}, \nu^{s}=0.2, c_{f}=0.3$ and $c_{1}=10.0 / \mathrm{J}^{-m^{-2}}$.

In Fig.4, mechanical responses in uniaxial compression predicted by three homogenization schemes are compared. It is shown that with the proposed damage criterion and friction condition, the dilute scheme and the PC-W scheme both lead to a material softening behavior. As commonly observed, the former is more brittle (sharp decrease of stress) than the latter. It seems that the interaction between microcracks induces more ductile response of material. Contrarily to these two schemes, the Mori-Tanaka estimate leads to a fully hardening behavior. In addition, the evolutions of the global damage value are provided in Fig.5, as functions of axial strain. 


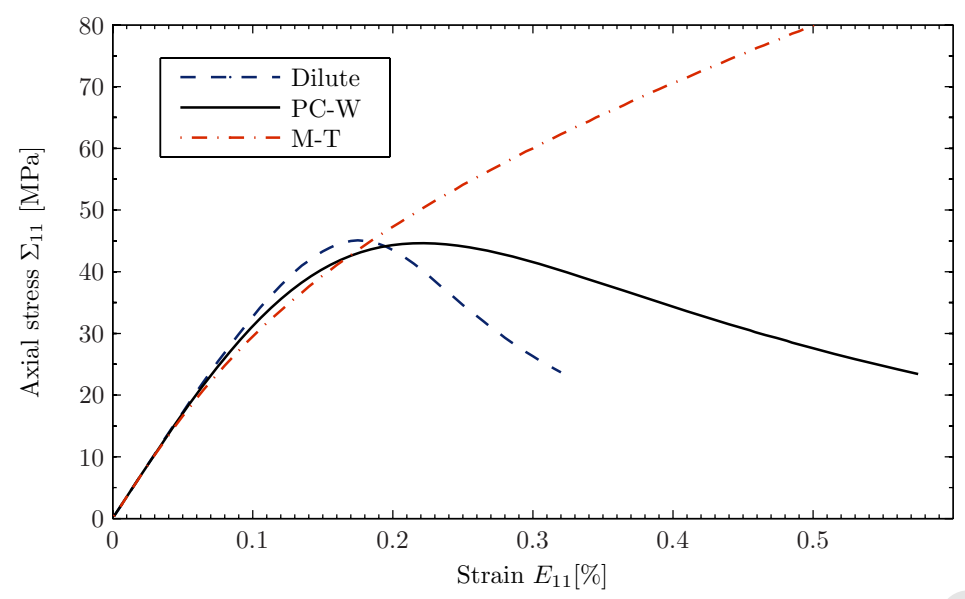

FIG. 4. Comparaison on predictions of mechanical responses for uniaixal compression test

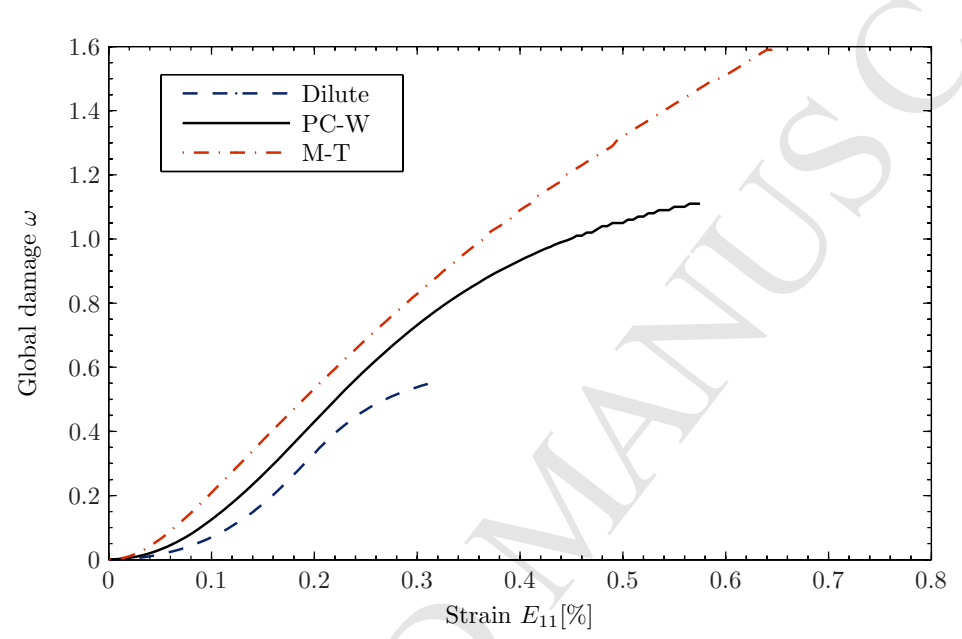

FIG. 5. Comparison on damage evolutions for uniaixal compression test

\subsection{Numerical predictions for cyclic triaxial compression}

The mechanical response and damage evolution predicted by the PC-W scheme for the cyclic loading path are illustrated in Fig. 6 and Fig. 7, respectively. The same values of parameters as those for the above monotonic tests have been used. From a qualitative point of view, we can observe three phases of response as in monotonous loading. Irreversible strains and hysteretic loops during loading - unloading reloading paths are observed as a consequence of the frictional mechanism. Further, open loops are found at the end of reloading path; this is in agreement with the evolution of the damage variable $\omega$ associated to the volumetric dilation.

\subsection{Numerical simulations for concrete tests}

Fig.8 and Fig.9 show comparisons between experimental results and numerical predictions respectively for uniaxial compression test and for compression test under plane-strain condition with kinematic constraint $E_{22}=0$. One can see that there is a good agreement for the axial stress-strain curves while the lateral strains are underestimated for both tests. This difference can be interpreted by the fact we have adopted here an associated flow rule for the determination of frictional sliding-induced inelastic deformation in the normal direction of cracks. The use of non-associated flow rule should improve this 


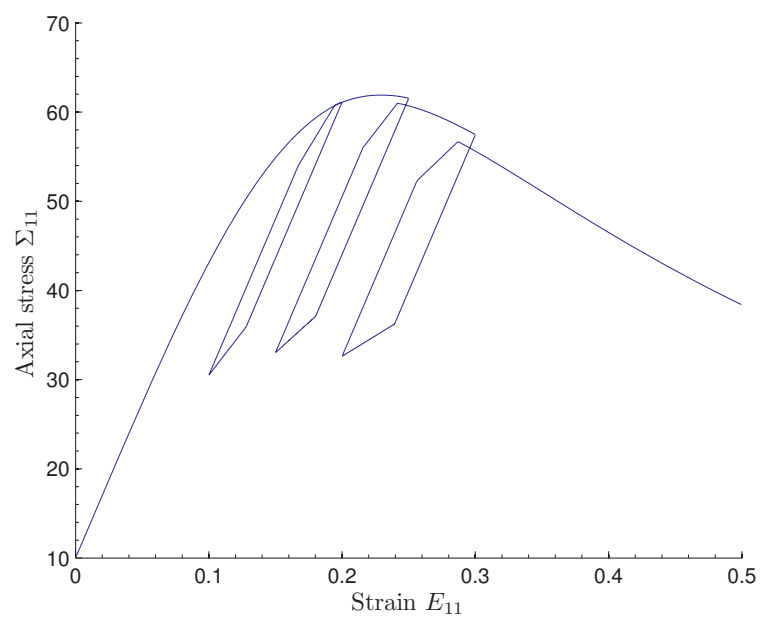

FIG. 6. Numerical prediction of mechanical response for cyclic compression loading with confining pressure $10 \mathrm{MPa}$

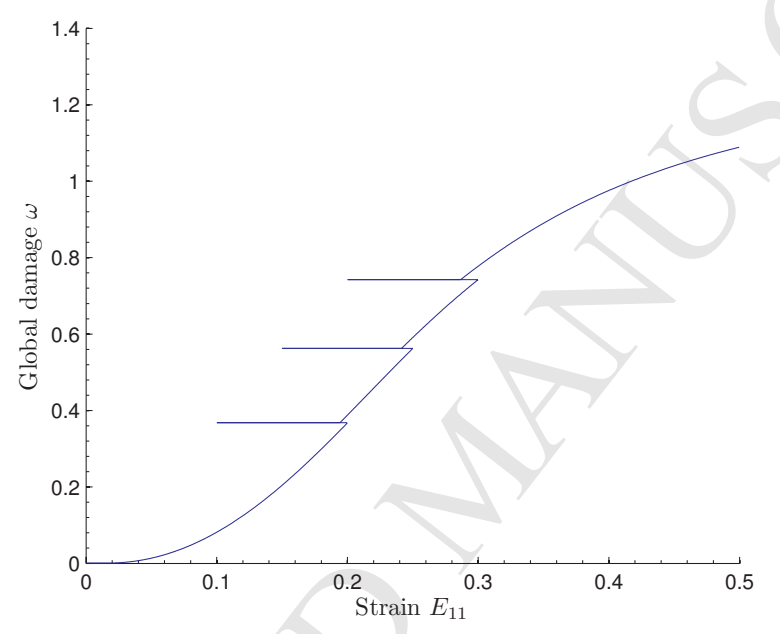

FIG. 7. Numerical prediction of damage evolution for cyclic compression loading with confining pressure $10 \mathrm{MPa}$ prediction.

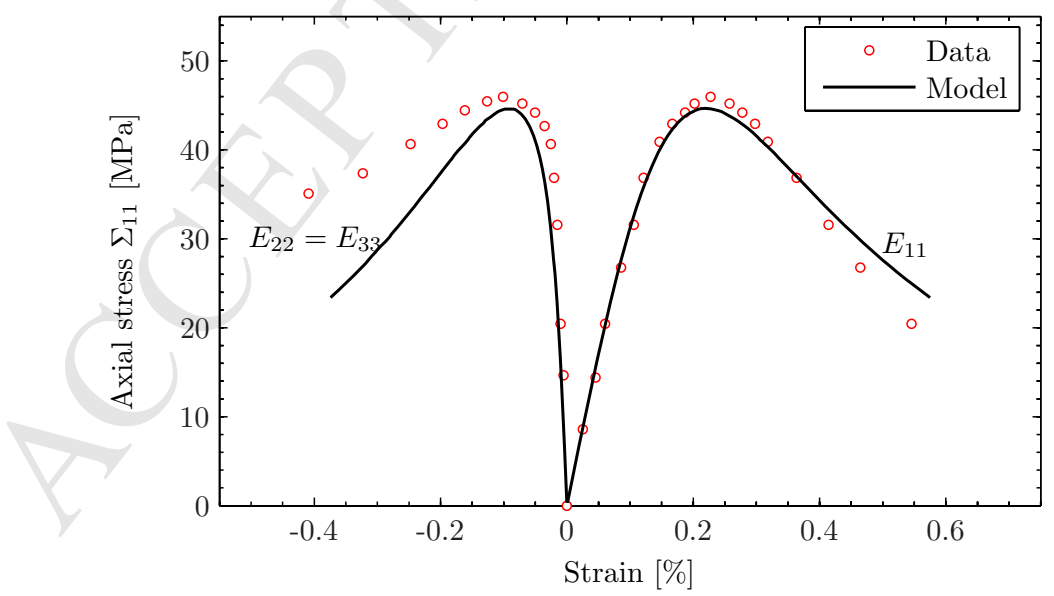

FIG. 8. Comparison between numerical prediction (PC-W scheme) and experimental data for unixial compression test 


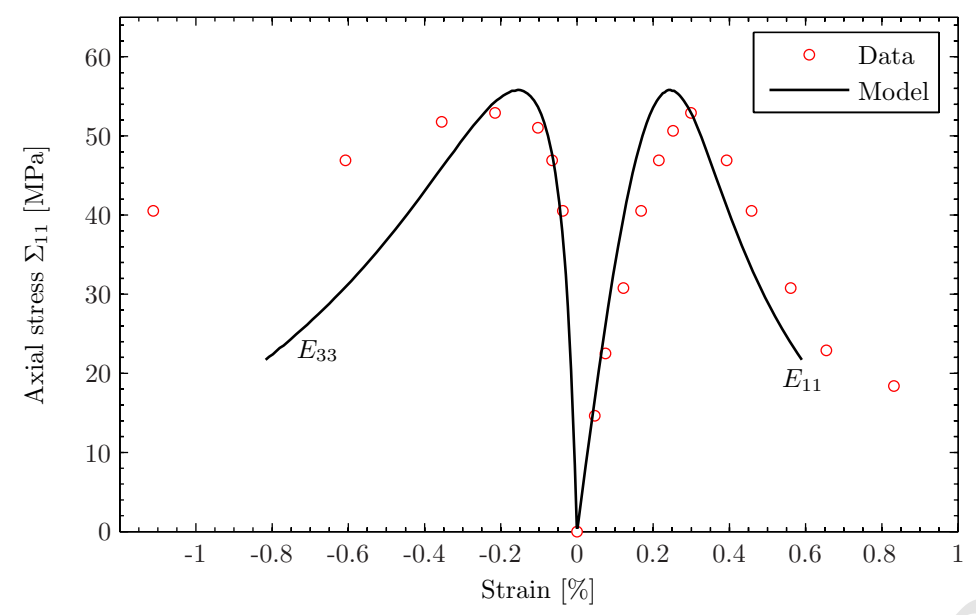

FIG. 9. Comparison between numerical prediction (PC-W scheme) and experimental data for compression test under plane strain condition

\section{Conclusions}

By applying the standard Eshelby-type homogenization procedure to the matrix - cracks system, an original homogenization-based formulation has been proposed for modelling coupled unilateral damage and friction-induced inelastic deformation in cracked brittle materials. Under isotropic simplification, constitutive equations for the damaged materials are established respectively for the open, closed frictionless and closed frictional cases. Within the thermodynamic framework, we have discussed some typical frictional yield conditions and treated in detail the coupling between damage and friction. It is concluded that the Von-Mises criterion can not satisfy the continuity conditions while the Coulomb criterion does. As examples of first phase validation, the proposed model was applied to simulate typical compression tests on concrete materials. The proposed model is able to reproduce the main features of mechanical behaviors observed in such class of brittle materials. Deeper validations will be necessary in order to more precisely determine the validity domains of the proposed model. Appropriate laboratory investigations, both on microscopic and microscopic scales will be also essential for the proper determination of model's parameters.

\section{Annexe A. Determination of $\mathbb{C}^{c}$ for closed frictionless microcracks}

Materials composed of an isotropic solid matrix and a family of parallel cracks exhibit transversely isotropic symmetry. The Walpole's tensorial algebra (Walpole, 1981) is proved particularly useful to carry out inner product of transversely isotropic tensors or tensor inversion, which is composed of a set of six direction-related fourth-order tensors $\mathbb{E}^{r}, r=1, \ldots, 6$. With notations $\boldsymbol{a}=\underline{n} \otimes \underline{n}$ and $\boldsymbol{b}=\boldsymbol{\delta}-\underline{n} \otimes \underline{n}$, the components $E_{i j k l}^{r}$ are then given as

$$
\left\{\begin{array}{l}
E_{i j k l}^{1}=\frac{1}{2} b_{i j} b_{k l}, \quad E_{i j k l}^{2}=\frac{1}{2} a_{i j} a_{k l}, \quad E_{i j k l}^{3}=\frac{1}{2}\left(b_{i k} b_{j l}+b_{i l} b_{j k}-b_{i j} b_{k l}\right), \\
E_{i j k l}^{4}=\frac{1}{2}\left(a_{i k} b_{j l}+a_{i l} b_{j k}+a_{j k} b_{i l}+a_{j l} b_{i k}\right), \quad E_{i j k l}^{5}=a_{i j} b_{k l}, \quad E_{i j k l}^{6}=b_{i j} a_{k l}
\end{array}\right.
$$

From these identities, it is shown that any transversely isotropic (not necessary symmetric) fourth-order tensor $\mathbb{U}$ can be expressed as a linear combination of these six elementary tensors $\mathbb{E}^{r}, r=1, \ldots, 6$ :

$$
\mathbb{U}=c \mathbb{E}^{1}+d \mathbb{E}^{2}+e \mathbb{E}^{3}+f \mathbb{E}^{4}+g \mathbb{E}^{5}+h \mathbb{E}^{6}
$$

which may be noted in the following symbolic form : 


$$
\mathbb{U}=(c, d, e, f, g, h)
$$

For the case that $\mathbb{U}$ possesses the diagonal symmetry, $g=h$, the coefficients are related to five independent elastic moduli

$$
c=2 k, \quad d-2 g^{2} / c=E_{a}, \quad g / c=\nu_{a}, \quad e=2 \mu_{t}, \quad f=2 \mu_{a}
$$

with $k, \mu_{t}, \mu_{a}, E_{a}, \nu_{a}$, all positive definite, as the plane strain bulk modulus, the transverse shear modulus, the axial shear modulus and the axial Young's modulus and Poisson's ratio, respectively.

When the solid matrix is weakened by one unique family of closed frictionless microcracks with the unit normal vector $\underline{n}$, which corresponds to the axis according to the specific penny-shaped geometry, the normal stress is assumed to be continuous across the microcrack faces. At the transition (from open to closed), the axial Young's Modulus, noted $E(\underline{n})=(\underline{n} \otimes \underline{n}):\left(\mathbb{C}^{\text {hom }}\right)^{-1}:(\underline{n} \otimes \underline{n})$ should return to its intact value $E^{s}$. Under the frictionless condition, the shear moduli $\mu_{t}$ et $\mu_{a}$ could be assumed to be unchange, i.e. zero-valued. The elastic modulus for microcracks $\mathbb{C}^{c}$ which as commonly, possesses diagonal symmetry, i.e. $g=h$, is then expressed in the Walpole's base as follows :

$$
\mathbb{C}^{c}=(c, d, 0,0, g, g)
$$

For the dilute scheme, the effective stiffness tensor $\mathbb{C}^{\text {hom }}$ is given by

$$
\mathbb{C}^{h o m}=\mathbb{C}^{s}-\varphi\left(\mathbb{C}^{s}-\mathbb{C}^{c}\right):\left[\mathbb{I}-\mathbb{S}_{\epsilon}:\left(\mathbb{I}-\left(\mathbb{C}^{s}\right)^{-1}: \mathbb{C}^{c}\right)\right]^{-1}
$$

We recall that $\varphi=\frac{4}{3} \pi \omega \epsilon$ and that a family of cracks characterized by its unit normal leads to a transversal isotropy in the material behavior. It is shown that there exist the following relations for the Eshelby tensor $\mathbb{S}_{\epsilon}$

$$
\mathbb{S}_{\epsilon}=\left(S_{1111}+S_{1122}, S_{3333}, S_{1111}-S_{1122}, 2 S_{1313}, S_{3311}, S_{1133}\right)
$$

with the components given in Mura (1987) and Nemat-Nasser and Hori (1993).

As commonly, the determination of $\mathbb{C}^{\text {hom }}$ first needs to calculate a limit, noted $\mathbb{Q}$

$$
\mathbb{Q}=\lim _{\epsilon \rightarrow 0} \epsilon\left[\mathbb{I}-\mathbb{S}_{\epsilon}:\left(\mathbb{I}-\left(\mathbb{C}^{s}\right)^{-1}: \mathbb{C}^{c}\right)\right]^{-1}
$$

After some tensorial calculations, $\mathbb{Q}$ is rewritten in the form :

$$
\mathbb{Q}=\left(\lim _{\epsilon \rightarrow 0} \frac{1-a_{2}}{l} \epsilon, \lim _{\epsilon \rightarrow 0} \frac{1-a_{1}}{l} \epsilon, \lim _{\epsilon \rightarrow 0} \frac{1}{1-a_{3}} \epsilon, \lim _{\epsilon \rightarrow 0} \frac{1}{1-a_{4}} \epsilon, \lim _{\epsilon \rightarrow 0} \frac{a_{5}}{l} \epsilon, \lim _{\epsilon \rightarrow 0} \frac{a_{6}}{l} \epsilon\right)
$$

with

$$
\left\{\begin{array}{l}
a_{1}=\left(S_{1111}+S_{1122}\right)\left[1-\frac{1}{E^{s}}\left(\left(1-\nu^{s}\right) c-2 \nu^{s} g\right)\right]-\frac{2}{E^{s}}\left(g-\nu^{s} c\right) S_{1133} \\
a_{2}=S_{3333}\left[1-\frac{1}{E^{s}}\left(d-2 \nu^{s} g\right)\right]-\frac{2}{E^{s}}\left(\left(1-\nu^{s}\right) g-\nu^{s} d\right) S_{3311} \\
a_{3}=S_{1111}-S_{1122}, \quad a_{4}=2 S_{1313} \\
a_{5}=S_{3311}\left[1-\frac{1}{E^{s}}\left(\left(1-\nu^{s}\right) c-2 \nu^{s} g\right)\right]-\frac{1}{E^{s}}\left(g-\nu^{s} c\right) S_{3333} \\
a_{6}=S_{1133}\left[1-\frac{1}{E^{s}}\left(d-2 \nu^{s} g\right)\right]-\frac{1}{E^{s}}\left(\left(1-\nu^{s}\right) g-\nu^{s} d\right)\left(S_{1111}+S_{1122}\right) \\
l=\left(1-a_{1}\right)\left(1-a_{2}\right)-2 a_{5} a_{6}
\end{array}\right.
$$

It is then proved that if and only if $d>0, \mathbb{Q}$ is non zero and takes the expression : 


$$
\mathbb{Q}=\frac{4}{\pi} \frac{1-\nu^{s}}{2-\nu^{s}} \mathbb{E}^{4}
$$

Consequently, (3) reduces to the form

$$
\mathbb{C}^{h o m}=\mathbb{C}^{s}:\left(\mathbb{I}-\frac{16}{3} \frac{1-\nu^{s}}{2-\nu^{s}} \omega \mathbb{E}^{4}\right)
$$

Note that $\omega$ denotes the damage variable. The homogenized compliance tensor $\mathbb{S}^{h o m}$ is obtained by inversing $\mathbb{C}^{\text {hom }}$

$$
\mathbb{S}^{h o m}=\left[\left(\mathbb{I}-\frac{16}{3} \frac{1-\nu^{s}}{2-\nu^{s}} \omega \mathbb{E}^{4}\right)\right]^{-1}: \mathbb{S}^{s}, \quad \mathbb{S}^{s}=\left(\mathbb{C}^{s}\right)^{-1}
$$

It is easily proved that $\mathbb{S}^{\text {hom }}$ satisfies $E(\underline{n})=E^{s}$.

As a conclusion, for the case of closed frictionless microcracks, we can take $\mathbb{C}^{c}=\kappa \underline{n} \otimes \underline{n} \otimes \underline{n} \otimes \underline{n}$ with $\kappa>0$ for modelling of mechanical behaviors.

\section{Références}

Andrieux, S., Bamberger, Y., Marigo, J., 1986. Un modèle de matériau microfissuré pour les roches et les bétons. Journal de mécanique théorique et appliquée 5(3), 471-513.

Barthelemy, J., Dormieux, L., Kondo, D., 2003. Détermination du comportement macroscopique d'un milieu à fissures frottantes. C.R. Mecanique 331, 7-84.

Budiansky, B., O’Connel, J., 1976. Elastic moduli of a cracked solid. Int. J. Solids Structures 12, 81-97.

Chiarelli, A., Shao, J., Hoteit, N., 2003. Modelling of elastoplastic damage behaviour of a claystone. International Journal of Plasticity 19, 23-45.

Deude, V., Dormieux, L., Kondo, D., Pensee, V., 2002. Conewise linear elastic materials. C.R. Mécanique 330, $587-592$.

Dormieux, L., Kondo, D., Ulm, F.-J., 2006. Microporomechanics. WILEY.

Dragon, A., Mroz, Z., 1979. A continuum model for plastic-brittle behaviour of rock and concrete. International Journal of Engineering Science 17, 121-137.

Eshelby, J., 1957. The determination of the elastic field of an ellipsoidal inclusion and related problems. Proc. R. Soc. Lond. A. 241, 375-396.

Fredrich, J., Evans, B., Wong, T., 1989. Micromechanics of the brittle to plastic transition in carrara marbe. J. Geophys. Res. 94, 4129-4145.

Gambarotta, L., Lagomarsino, S., 1993. A microcrak damage model for brittle materials. Int. J. Solids Structures 30(2), $177-198$

Hayakawa, K., Murakami, S., 1997. Thermodynamical modeling of elastic-plastic damage and experimental validation of damage potential. International Journal of damage mechanics 6, 333-363.

$\mathrm{Ju}, \mathrm{J} ., 1989$. On energy based coupled elastoplastic damage theories : constitutive modeling and computational aspects. Int. J. Solids Structures 25(7), 803-833.

Krajcinovic, D., 1996. Damage mechanics. North-Holland, Amsterdam, The Netherlands.

Mori, T., Tanaka, K., 1973. Averages stress in matrix and average elastic energy of materials with misfitting inclusions. acta metall. 21, 571-574.

Mura, T., 1987. Micromechanics of defects in solids, 2nd edition. Martinus Nijhoff Publ., The Hague, Boston.

Nemat-Nasser, S., Hori, M., 1993. Micromechanics : overall properties of heterogeneous materials. North-Holland, Amsterdam, The Netherlands.

Pensee, V., Kondo, D., Dormieux, L., 2002. Micromechanical analysis of anisotropic damage in brittle materials. J. Engng. Mech., ASCE 128(8), 889-897.

Ponte-Castaneda, P., Willis, J.-R., 1995. The effect of spatial distribution of effective behavior of composite materials and cracked media. Journ. Mech. Phys. Solids 43, 1919-1951.

Shao, J.-F., 1998. Poroelastic behaviour of brittle rock material with anisotropic damage. Mechanics of Materials 30 (1), $41-53$.

Shao, J.-F., Jia, Y., Kondo, D., Chiarelli, A.-S., 2006. A coupled elastoplastic damage model for semi-brittle materials and extension to unsaturated conditions. Mechanics of Materials 38 (3), 218-232.

Van Mier, J., 1984. Strain-softening of concrete under multiaxial loading conditions. Ph.D. thesis, Technishe Hogeschool Eindhoven. 
Walpole, L., 1981. Elastic behavior of composite materials : theoretical foundations. Advances in Applied Mechanics, ed. C. S. Yih,Vol. 21, 169-242.

Wong, T., 1982. Micromechanics of faulting in westerly granite. International Journal of Rock Mechanics and Mining Sciences $19,49-62$.

Zaoui, A., 2002. Structural morphology and constitutive behavior of microheterogeneous materials. Dans : Continuum micromechanics, Ed. P. Suquet, Springer, New-York, 291-347.

Zhu, Q.-Z., Kondo, D., Shao, J.-F., 2008a. Micromechanical analysis of coupling between anisotropic damage and friction in quasi brittle materials : role of the homogenization scheme. International Journal of Solids and Structures 45(5), $1385-1405$.

Zhu, Q.-Z., Kondo, D., Shao, J.-F., Pensee, V., 2008b. Micromechanical modelling of anisotropic damage in brittle rocks and application. International Journal of Rock Mechanics and Mining Sciences 45, 467-477. 